\title{
Estudio de normativas urbano-edilicias para la eficiencia energética. Godoy Cruz, Mendoza, Argentina.
}

\author{
Dana Otero ${ }^{1}$, Ricardo Cohn $^{2}$, Mariela Arboit ${ }^{3}$ \\ INCIHUSA CCT CONICET Mendoza. Mendoza, Argentina \\ E-mail: ${ }^{1}$ dotero@mendoza-conicet.gob.ar, ${ }^{2}$ rcohn@mendoza-conicet.gob.ar, \\ 33marboit@mendoza-conicet.gob.ar
}

\begin{abstract}
Resumen. El crecimiento poblacional y el consumo de energías no renovables, afectan los recursos ambientales de los que dependen los habitantes urbanos. La temática ha sido trabajada en numerosas investigaciones y muchos países han implementado estrategias de eficiencia energética a través de los códigos de edificación logrando acciones exitosas.

El trabajo analiza la normativa urbano-edilicia en el Área Metropolitana de Mendoza (AMM), conformada por 6 municipios. Metodológicamente se realizó un relevamiento de datos, análisis, actualización de información y elaboración de cartografía GIS para el municipio de Godoy Cruz y posteriormente se realizó un análisis comparativo preliminar de zonificación, indicadores y estrategias urbanas sostenibles en los códigos vigentes para los restantes municipios.

La investigación permitió cartografiar y cuantificar unificadamente las distintas normativas intervinientes, evidenciando diferencias en las variables analizadas en el AMM y escasez de indicadores energéticos en la mayoría de los municipios. Los resultados del análisis muestran que Guaymallén, Ciudad de Mendoza y Godoy Cruz, son los municipios con mayor cantidad de indicadores urbanoedilicios, seguidos de Maipú y Luján de Cuyo. En Godoy Cruz la normativa permite predominantemente de bajas a medias densidades residenciales, donde los indicadores urbano-edilicios son más favorables para densificar zonas comerciales y administrativas, quedando pendiente el análisis para los demás municipios. Godoy Cruz y Ciudad de Mendoza han desarrollado las primeras acciones tendientes a regular la eficiencia energética en edificios.

El objetivo futuro consiste en proponer estrategias y lineamientos a fin de conformar un Código de Eficiencia Energética para el AMM.
\end{abstract}

Palabras clave: normativa urbana y edilicia, consumo energético, eficiencia energética.

\section{Introducción}

A nivel internacional se muestran acciones de desarrollo, implementación y cumplimiento de regulaciones de eficiencia energética en edificios para países emergentes y desarrollados (Iwaro y Mwasha, 2010). Se han implementado objetivos verdes en los códigos de construcción, para la conservación de recursos y la reducción de desechos (APEC, 2013). Se han logrado transiciones exitosas hacia energías renovables mediante la implementación en códigos nacionales de construcción (Enker y Morrison, 2017). Coexisten hoy diversos sistemas de regulación (Pedro, Meijer y Visscher, 2010) como también dificultades y falencias (Pan y Garmston, 2012), con un escenario internacional activo frente a la problemática.

En Argentina, la mayor parte de la energía utilizada proviene de fuentes de energía no renovable, térmica con el $64,75 \%$ e hidráulica con el 30,09\% (CAMMESA, 2019a). 
Observándose un aumento sostenido del consumo anual de energía, superando en 2018 el máximo histórico de invierno (CAMMESA, 2019b), se han implementado acciones para mejorar la provisión y producción de energías renovables (Ley Nacional 27.191, 2015). A escala municipal Rosario fue pionero incorporando aspectos higrotérmicos y de demanda energética de forma escalonada desde 2011 (Ordenanza 8.757, 2011). Aún queda pendiente incorporar soluciones en la mayoría de los códigos vigentes en el resto de los municipios de las provincias.

En la Provincia de Mendoza, la generación de energía a partir del recurso hídrico representa el 60,5\% (EMESA, 2019). De los datos energéticos de la provincia de Mendoza, el consumo de gas natural provincial total se divide en tres: $26 \%$ correspondiente a uso doméstico, 4\% a comercial y servicios y el $70 \%$ restante pertenece a los sectores: central eléctrica, transporte, industrial, GNC y subdistribuidoras. Del total de consumos doméstico, comercial y servicios $(30 \%)$ en el AMM; el $12 \%$ corresponde a comercial y servicios y el $88 \%$ a doméstico. Godoy Cruz tiene una participación del $21 \%$ del consumo doméstico mensual del AMM (Morales, Arboit y Cucchietti, 2017). Respecto de la energía eléctrica el consumo total anual en 2017

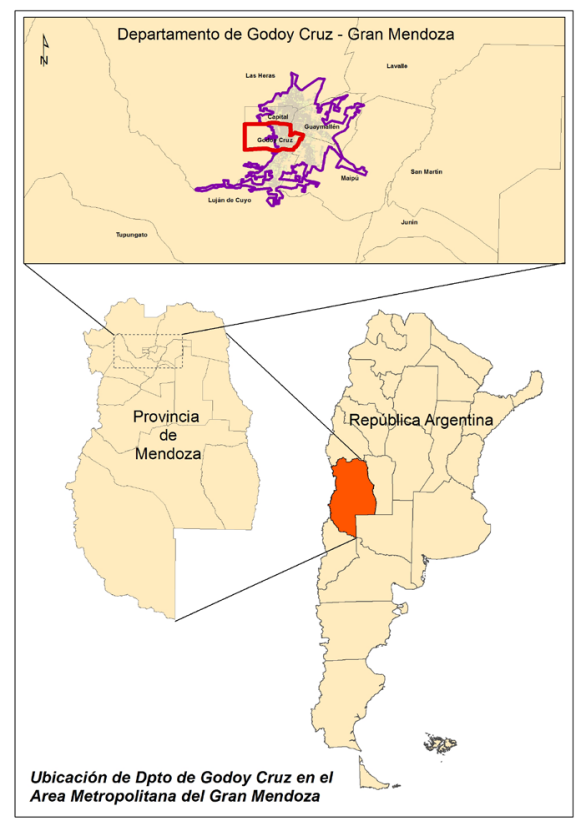

Fig. 1. Ubicación del caso de estudio para Godoy Cruz fue de 343.558.664 kWh, donde el $55 \%$ correspondió para el sector residencial con $187.518 .221 \mathrm{kWh}$, el $13 \%$ para el comercial con $45.526 .217 \mathrm{kWh}, 25 \%$ para el industrial con $86.734 .177 \mathrm{kWh}$ y el $7 \%$ para el alumbrado público con $23.780 .049 \mathrm{kWh}$ (Ordenanza 6.876, 2018).

El objetivo del presente trabajo es evidenciar las distintas normativas de morfología urbanoedilicia en los municipios del AMM, tomando como caso de estudio individual al municipio de Godoy Cruz; para analizar en forma comparativa lo implementado respecto de: zonificación, indicadores urbano-edilicios y estrategias urbanas tendientes a un desarrollo sostenible.

\section{Antecedentes}

Caso de estudio

El AMM, está ubicada en el centro-oeste argentino, presenta clima mesotermal árido con inviernos templado-fríos, veranos cálido-secos e intensa radiación solar (media anual: 18,06 $\mathrm{Mj} / \mathrm{m} 2$ día). Horas anuales en confort: $21,53 \%$, calefacción necesaria: 70,14\% y enfriamiento necesario: $8,33 \%$.

El caso de estudio, Godoy Cruz, es uno de los 6 municipios del AMM. Su posición geográfica es $32^{\circ} 54^{\prime}-32^{\circ} 59^{\prime}$ Latitud Sur y $68^{\circ}$ 45'- $69^{\circ} 12^{\prime}$ Longitud Oeste. Altitud media: 859 m.s.n.m. Cuenta con una población de 189.578 hab.

Godoy Cruz tiene una superficie departamental de $107.3 \mathrm{~km} 2$, organizada en 6 distritos (Fig. 2). Del total de hogares 57.375, el $17 \%$ son deficitarios (INDEC, 2010), en 2015 existían 202 barrios formales y 10 asentamientos informales (Fig. 3). En 2018 los asentamientos ascienden a 12 (Ordenanza 6.876, 2018), algunos continúan existentes, otros son nuevos, y otros se convirtieron en barrios formales.

\section{Estudio Normativo Macro}

Los códigos urbano-edilicios son normativas regulatorias del espacio público y privado determinando la morfología de la ciudad, mediante sus indicadores. Surgen en 

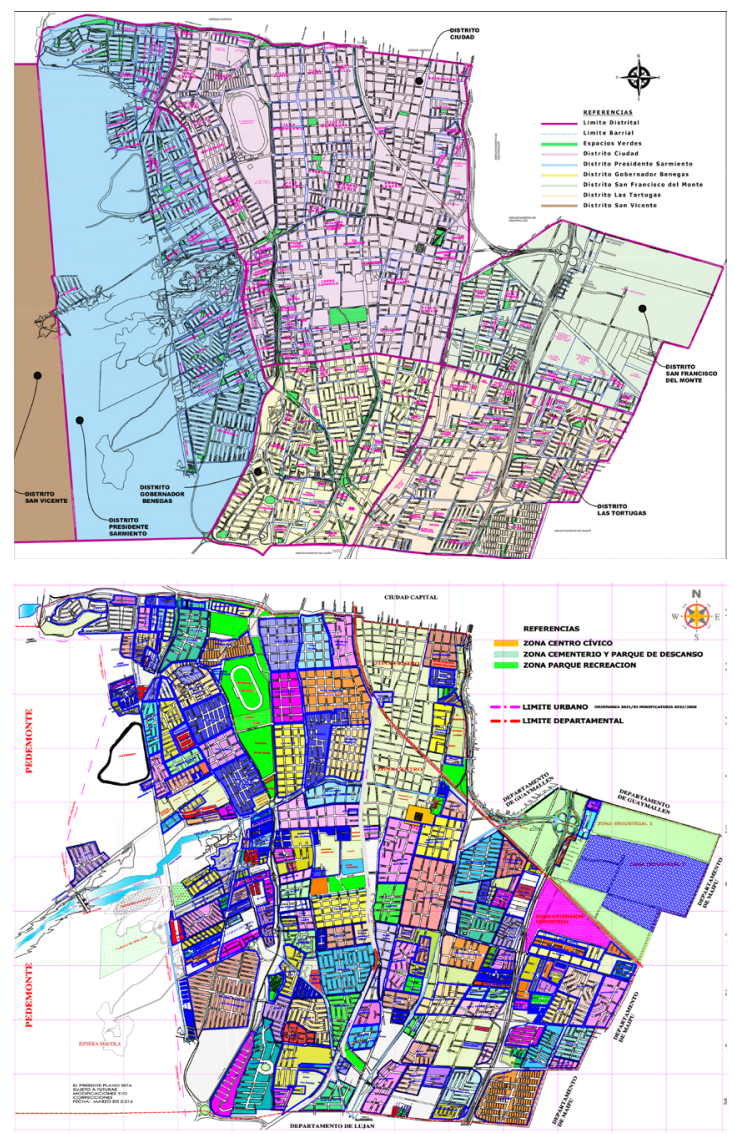

Fig. 2. (arriba) Plano con Distritos y Barrios 2019. Fuente: Municipalidad de Godoy Cruz.

Fig. 3. (abajo) Plano con Barrios 2015. Fuente: Municipalidad de Godoy Cruz.

Mendoza en los '70 con parámetros básicos de regulación territorial. En 1983 se dispone el uso del suelo en el oeste del gran Mendoza (Ley Provincial 4.886, 1983) y desde 2017 rige el Plan Provincial de Ordenamiento Territorial (Ley Provincial 8.999, 2017 - PPOT) a fin de orientar la política pública y los diferentes planes de ordenamiento. El PPOT es una oportunidad para los municipios de generar cambios en normativas y códigos.

\section{Estudio Normativo Micro del Municipio de Godoy Cruz}

El municipio se divide en dos grandes áreas: piedemonte sin urbanizaciones con 71.3 $\mathrm{km} 2$ y urbanizada (planicie + piedemonte) con $36 \mathrm{~km} 2$. El área urbanizada de planicie, conformada por cinco de los seis distritos geográficos: Ciudad, Gobernador Benegas,
San Francisco del Monte, Las Tortugas y parte de Presidente Sarmiento (Fig. 4). Está regida por la ordenanza $4.947 / 03$, que establece la zonificación y usos del suelo; y la ordenanza 5.924/11, que define Indicadores Urbanos y microzonificación residencial.

En el área urbanizada del piedemonte se superponen las normativas municipales con la Ley Provincial 4.886/83, que determina 13 zonas y disposición del suelo hacia el oeste del Gran Mendoza; que propone criterios de preservación medioambiental, determina usos, dimensiones de lotes e indicadores de ocupación y construcción máxima para dos usos: urbanización parques y clubes de campo (Tablas 1 y 2). Dentro de los límites y zonificaciones que impone la Ley, a Godoy Cruz le corresponden las Zonas de la 9 a la 13, ubicadas en los distritos Presidente Sarmiento, Ciudad y Gobernador Benegas (Fig. 5 y Tabla 3). Dichas zonas compuestas mayoritariamente por proyectos de viviendas sociales. En los sectores correspondientes a la Ley aún sin urbanizaciones, los proyectos con terrenos mayores a $1000 \mathrm{~m} 2$ son elevados a la "Comisión de Piedemonte" para que dictamine.

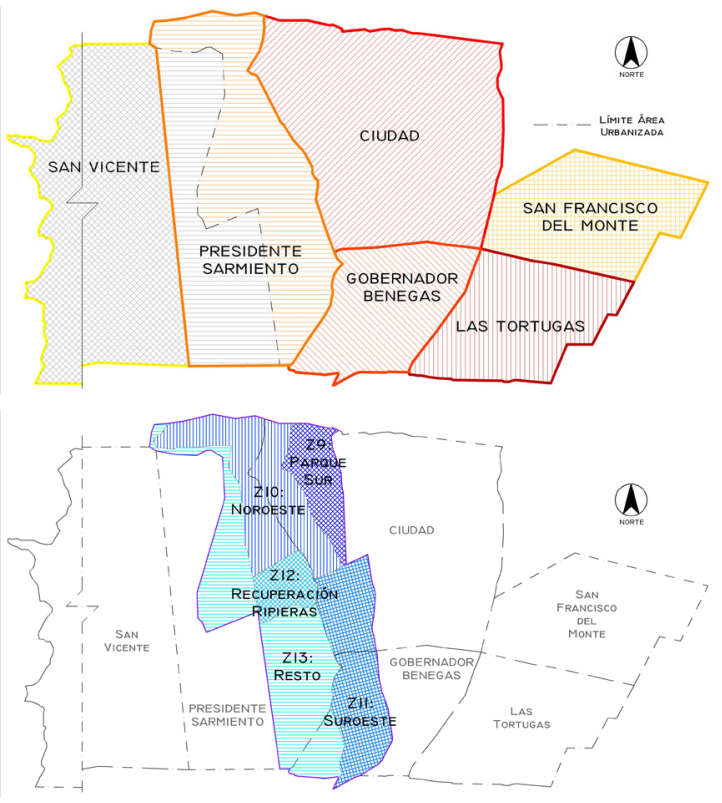

Fig. 4. (arriba) Distritos Urbanizados.

Fig. 5. (abajo) Zonas Ley 4886/83. 


\section{Metodología}

Se analizaron los antecedentes del marco normativo influyente en el AMM. Se profundizó, en primera instancia, el caso de estudio del municipio de Godoy Cruz mediante el relevamiento de datos (Municipal de Godoy Cruz, 2019 y Legislatura de Mendoza, 2019), análisis detallado y actualización de la información. Posteriormente se confeccionó la cartografía en formato GIS; se trabajó sobre la base de cartografía digital del municipio en formato dwg (AutoCAD) y formato shapefile (ESRI) del Instituto Geográfico Nacional (IGN), SIG 250 (2015). Se georreferenció y cambió de formato los dwg a shapefile, ajustándolos a la cartografía de base del IGN y/o imágenes satelitales de servidores públicos (Google Maps, etc.). Se incorporó la información de las ordenanzas. Posteriormente, se contrastó la información y se realizaron ajustes finales.

En una segunda etapa se implementó la metodología desarrollada en el resto de los municipios del AMM; se realizó un análisis comparativo preliminar de los códigos urbanos y de edificación de los 6 municipios, haciendo foco en la identificación de zonas e indicadores (Tabla 4), con especial interés en las estrategias vigentes para un desarrollo urbano sostenible.

\section{Resultados}

Análisis de Godoy Cruz

\section{Zonas}

Se analiza en el trabajo la zonificación residencial por su representatividad dentro del área urbana.

La cartografía de zonificación elaborada por el municipio en 2003 (Fig. 6) y actualizada en 2011 establece una microzonificación de la zona residencial (Fig. 7). Metodológicamente la información relevada fue procesada, reagrupando las zonas según sus características primarias en: Residencial (R); Residencial Especial; Residencial Mixta (RM); Comercial Mixta (CM); Comercial (C) y Especial (Tabla $5)$.

Las zonas primarias no contempladas en este trabajo, que representan el $20 \%$ del

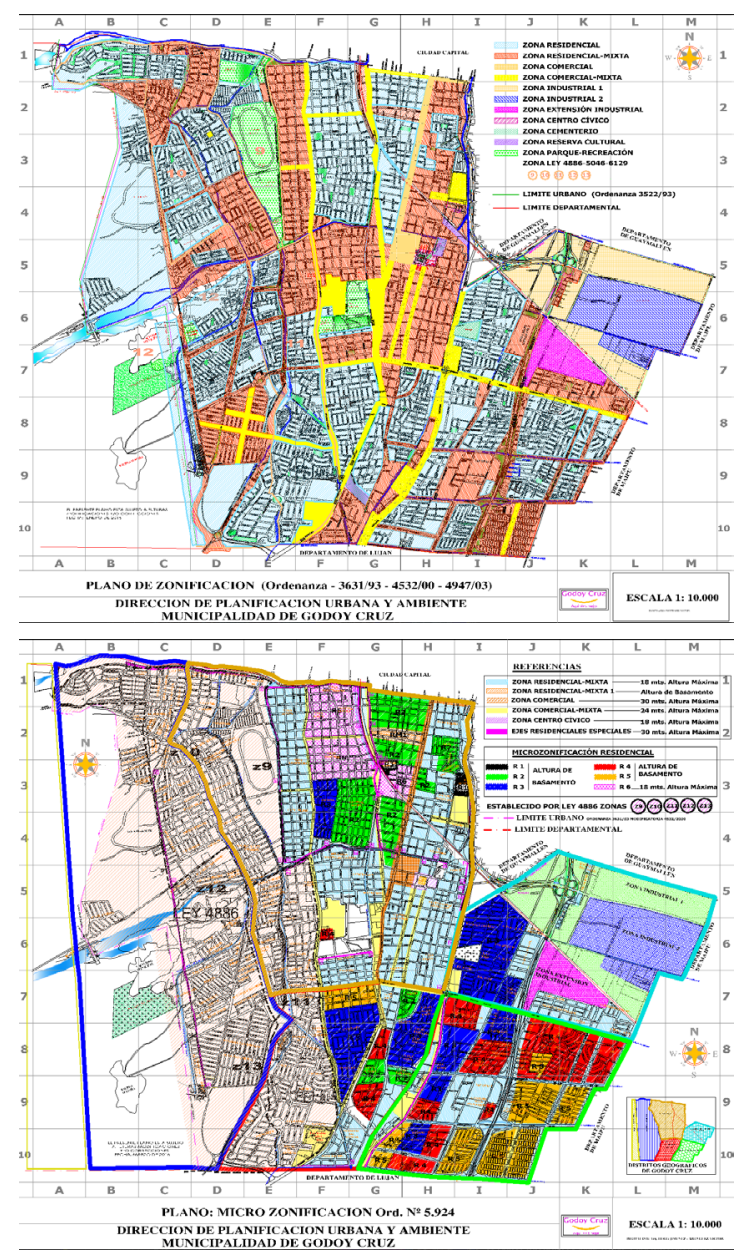

Fig. 6. (arriba) Zonificación s/ Ord. 4.947/03. Fuente: Municipalidad de Godoy Cruz.

Fig. 7. (abajo) Zonificación s/Ord. 5.924/11. Fuente: Municipalidad de Godoy Cruz.

área de estudio y requieren de normativas específicas, fueron: Industrial (sin indicadores de relevancia), Recreación y Piedemonte sin urbanizaciones.

Actualmente el municipio no cuenta con la información cartografiada de la microzonificación propuesta en la ordenanza 5.924/11, debiendo remitirse al mapeo de barrios (Fig. 2 y Fig. 3).

En el presente trabajo se elaboró la cartografía en GIS, considerando la microzonificación, esto permite su georreferenciación, actualización e identificación de cada una de las morfologías urbano-edilicias para elaborar, a futuro, estrategias de ahorro energético asociado a las normativas municipales.

Con la cartografía elaborada, se pudo realizar una cuantificación de superficies por 
zonas primarias y microzonificación (Fig. 8).

Los Ejes Residenciales Especiales, han sido considerados según su longitud totalizando aproximadamente $19554 \mathrm{~m}$.

El análisis de resultados arroja un municipio fuertemente residencial con un $80 \%$ de áreas $\mathrm{R}$ y RM. Dentro de la zona R (51\%) se distingue la microzonificación propuesta por la Ordenanza $5.924 / 11$ (de R1 a R6), con el $43 \%$, y el $8 \%$ restante corresponde a la zona residencial designada por la Ordenanza 4.947/03 sin microzonificar (Residencial) la cual no presenta indicadores y ocupa aproximadamente 284,5 ha. Los porcentajes más altos de $\mathrm{R}$ corresponden a: R5 13\% (barrios formales, asentamientos informales y consolidados) y R3 12\% (barrios formales).

\section{Indicadores en Godoy Cruz}

Los indicadores vigentes son: Densidad Poblacional, Tamaño de Parcela, FOS, FOT, h basamento, Hmáx y Retiros (tabla 4), se muestran según las zonas en tabla 5 . Se observa que las zonas con densidades bajas (R1 a R5 y RM1) presentan valores iguales en sus indicadores, mientras que las zonas con densidades medias poseen distintos valores de indicadores.

La densidad poblacional propuesta según zonas es: baja (R1, R2, R3, R4, R5 y RM1), media (R6, Ejes Residenciales Especiales, RM, Centro Cívico y CM) y alta (C). Siendo predominantes en un $99 \%$ la baja y media densidad.

El tamaño mínimo para lote urbano $(100 \mathrm{~m} 2)$ es coincidente con la Ley Provincial $4.341 / 79$ y no presenta regulación de forma. Respecto de FOS y FOT, los valores más elevados corresponden a zonas comerciales, institucionales y parcelas pequeñas. El basamento, depende del ancho de calzada (entre eje de calle y LM) y es optativo, siendo su altura máxima $12 \mathrm{~m}$ y $7 \mathrm{~m}$ en pasajes.

El indicador Hmáx vigente según zonas es: 30m (Ejes Residenciales Especiales y C), 24m (CM) y $18 \mathrm{~m}$ (R6, RM y Centro Cívico). En sectores de baja densidad (R1, R2, R3, R4, R5 y RM1) la Hmáx corresponde a la h basamento. La Hmáx está relacionada al FOT, ya que la limita según el tamaño de la parcela.

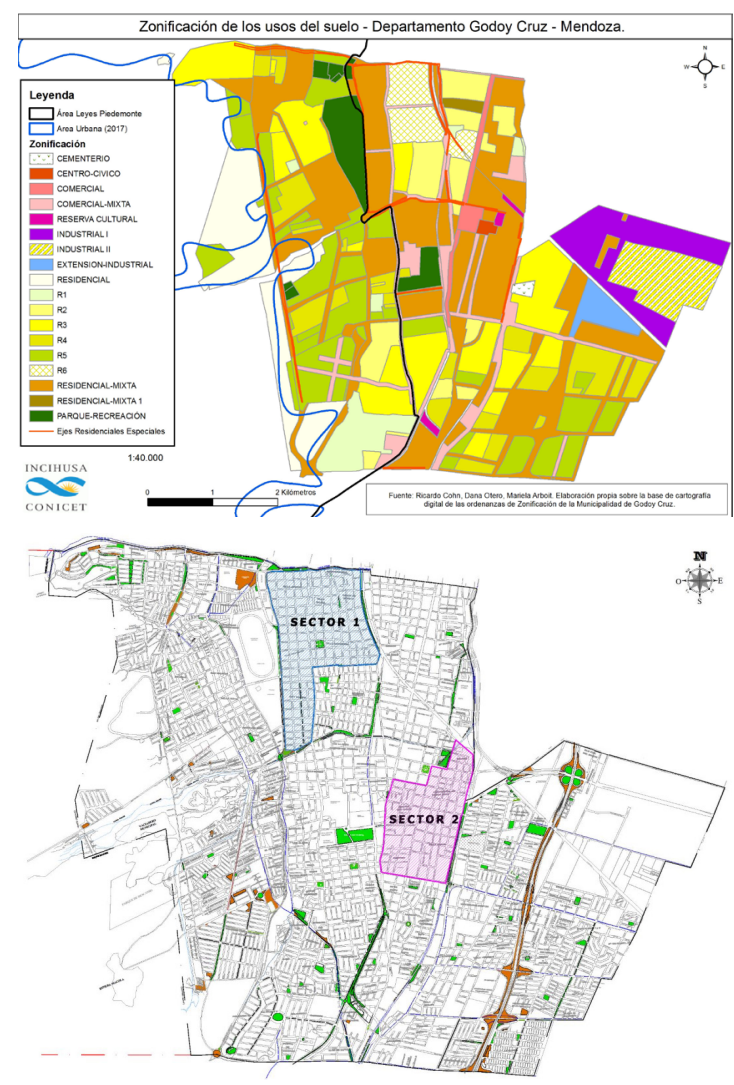

Fig. 8. (arriba) Zonificación actualizada de Godoy Cruz en formato GIS.

Fig. 9 (abajo) Ubicación Sector 1 y 2.

En relación con los retiros: Frontal (RF), Lateral (RL), Posterior (RP) y Separación de Torres, dichos indicadores son similares en todas las zonas (retiro mínimo $=5 \mathrm{~m}$ ), con excepción de los Ejes Residenciales Especiales (Tabla 5).

Con relación a los indicadores (Hmáx, FOS y FOT) es posible aumentarlos (Ordenanza 6.589, 2016 y Ordenanza 6.613, 2016), mediante una contribución compensatoria del $20 \%$ del costo de la superficie incrementada, destinado a obras de carácter urbano a especificar por el Municipio; en sectores de: hmáx basamento 9m (Tabla 7), sector 1 y 2 (Fig. 9 y Tabla 8 ) y Ejes Residenciales Especiales (Tabla 5), donde en estos últimos también se modifican los retiros.

\section{Análisis preliminar del Área Metropolitana de Mendoza.}

El análisis de los resultados generales del 


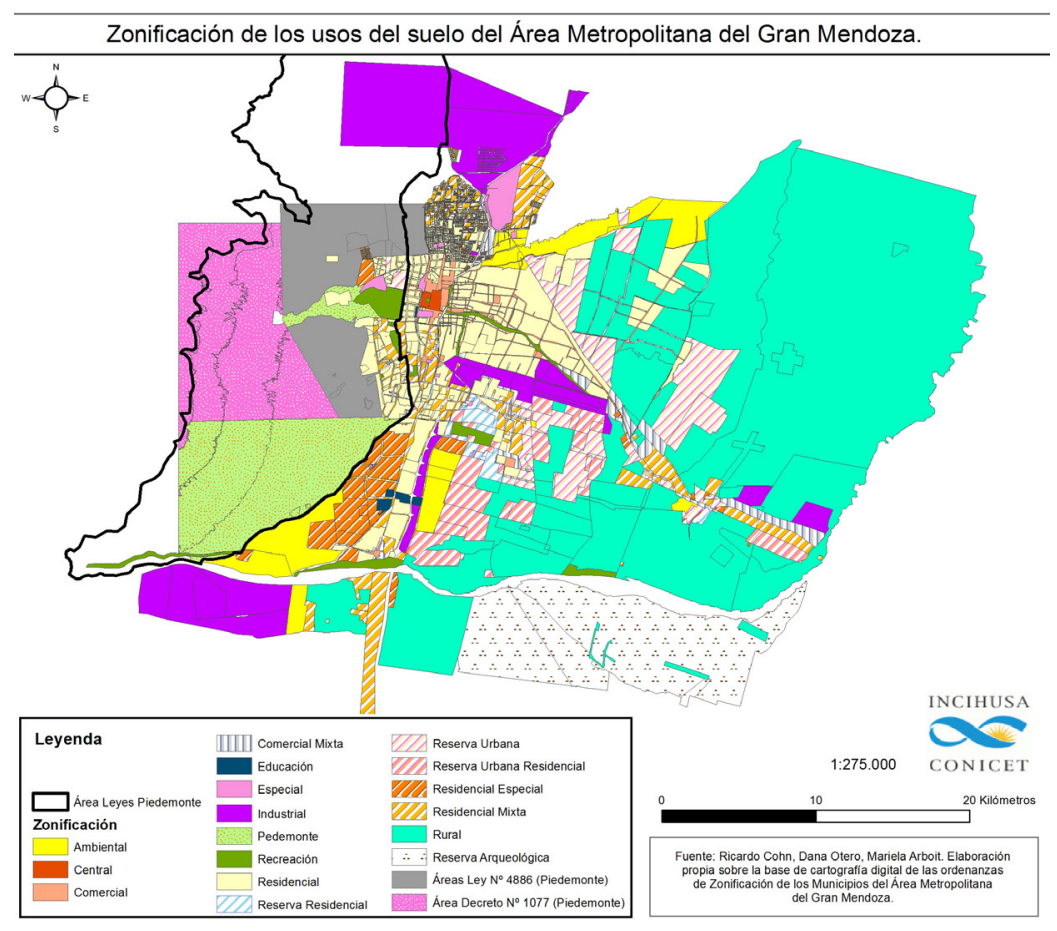

Fig. 10. Zonificación Primaria del AMM.

AMM permite visualizar comparativamente zonas exclusivas y similares entre municipios (Tabla 9), esta similitud nominativa no implica que compartan valores e indicadores.

Con la información relevada y procesada se realizó la cartografía preliminar GIS de la zonificación vigente en los 6 municipios del AMM (Fig. 10). Como también se confeccionaron tablas generales preliminares, agrupando en zonas primarias, zonas y subzonas (Tabla 9).

En la Tabla 10 se muestra un resumen comparativo de los indicadores del AMM por municipio respecto a la zonificación expuesta en la Tabla 9. De la comparación departamental de indicadores, Godoy Cruz y Ciudad de Mendoza incorporaron indicadores de desarrollo urbano sustentable en sus normativas (Tabla 11). Siendo estos: Corazón de Manzana, Construcciones Sustentables y Energías Limpias.

Corazón de Manzana: Se refiere al espacio libre en predio privado de suelo absorbente, $4 \mathrm{~m}$ mínimo entre fachada de fondo y límite posterior, corresponde al $60 \%$ del FOS restante. En casos excepcionales no es aplicable. En Ejes Residenciales Especiales se solicita el $80 \%$ del FOS restante de suelo absorbente con riego eficiente y vegetación de bajo consumo hídrico (Ordenanza 6.134, 2012).

Construcciones Sustentables: es incorporado por los municipios de Godoy Cruz y Ciudad de Mendoza. Godoy Cruz, propone beneficios en aforo y tasa municipal (Tablas 12 y 13) a proyectos edilicios nuevos o existentes, públicos o privados; que reduzcan el impacto medioambiental. Aplicando estrategias de diseño bioclimático, materiales o tecnología. Afectados con recargos en caso de no cumplimiento (Ordenanza 6.538, 2016 y Ordenanza 6.646, 2017). Ciudad de Mendoza, tiene exigencias mínimas para todos los proyectos (Tabla 14). Los proyectos se evalúan según parámetros (Tabla 15) y sub parámetros de sustentabilidad debiendo cumplir 3 de 5, para adquirir el beneficio de incremento de FOT, del $5 \%$ al $25 \%$ según tamaño de proyecto y categoría de la $\mathrm{A}$ (máximo beneficio) a la $\mathrm{F}$ (sin beneficio, mínimo de obligatoriedad). El incumplimiento es multado según valor de $\mathrm{m} 2$ construido no cumplido y sin obtención de Certificado Conforme a Obra (Ordenanza 3.944, 2018).

Energías Limpias: Crédito con tasa preferencial para la compra de equipos de energía renovable para viviendas familiares; micro y pequeñas empresas. Las cuotas se bonifican a la empresa eléctrica de Godoy 
Cruz. El programa se encuentra paralizado desde octubre 2018. Desde 2016 se registraron alrededor de 200 beneficiarios de viviendas particulares (Ordenanza 6.664, 2017).

\section{Conclusiones}

La investigación ha permitido el análisis de normativas urbano-edilicias en la totalidad de los municipios del AMM, profundizando el análisis para el municipio de Godoy Cruz. La identificación de zonificación e indicadores permitió un análisis comparativo preliminar entre municipios, evidenciando que Godoy Cruz y Ciudad de Mendoza son pioneros en la implementación de estrategias tendientes a un desarrollo urbano sostenible.

Godoy Cruz presenta un carácter residencial fuerte con el $60 \%$ de la población concentrada en dos distritos: Ciudad y Presidente Sarmiento (Ordenanza 6.876, 2018), parte de los mismos pertenecen al sector de superposición con la Ley Provincial 4.886/83. Es el único municipio del AMM que tiene en vigencia el indicador densidad poblacional, observándose en su área urbana un promedio más bajo que el pretendido por el municipio, cercano a 56hab/ha. Con relación a ello y respecto de FOS y FOT, se observa una intencionalidad de aumento en la densificación en zonas con usos comerciales, institucionales y en parcelas pequeñas.

Respecto del indicador corazón de manzana, es muy valioso para conservar superficies permeables de suelo a fin de compensar la huella edilicia en el municipio, dicho indicador no tiene una prosecución cuando la obra finaliza por lo que sería necesario un procedimiento de seguimiento para asegurar el cumplimiento de este y garantizar el beneficio que aporta.

El programa construcciones sustentables, bonifica según el tamaño de la obra, sin ponderar el aporte real que genera la estrategia bioclimática implementada. Ofrece mayores beneficios a las obras de mediana envergadura, siendo las que más se están desarrollando en el municipio. Es factible en su implementación, aunque el beneficio económico propuesto por el municipio no acompaña la vida útil de la construcción. Si bien es un buen inicio para concientizar a la industria de la construcción, sería necesario que contemplara el aporte ambiental generado al momento de la bonificación.

Godoy Cruz, es el único municipio del AMM que ha implementado un programa de energía limpias.

La implementación de normativas obligatorias tendientes a un desarrollo urbano más eficiente requerirá del conocimiento y adaptación de dichas normativas a la realidad compleja.

El presente trabajo ha elaborado una base de datos georreferenciados en formato GIS; que estará disponible para los responsables y planificadores urbanos.

El estudio comparativo preliminar del AMM de zonas e indicadores por municipio permitió visualizar que los códigos urbano-edilicios funcionan en forma individual, situación reflejada en la zonificación, sin una visión de conjunto en problemáticas que involucra al AMM, incluyendo a aquellas referidas a la eficiencia energética del sector residencial.

Los municipios más completos en normativa urbano-edilicia son Guaymallén, Ciudad de Mendoza y Godoy Cruz. Los dos últimos, si bien presentan solapamientos normativos en la zona pedemontana, son los más ordenados en cuanto a disponibilidad de información y legislación, y han implementado indicadores de desarrollo urbano sostenible.

La investigación ha evidenciado la necesidad de proponer cambios normativos unificados para toda el AMM, tendientes a mejorar la eficiencia energética edilicia según las diferentes morfologías existentes y las condicionantes climáticas propias. Esto podría implementarse en forma conjunta a través de un Código de Eficiencia Energética para el AMM.

El trabajo futuro plantea implementar la presente metodología de análisis detallado, realizado para Godoy Cruz, al resto de los municipios, a fin de proponer estrategias de eficiencia energética a implementar por medio de las normativas a la totalidad del Área Urbana Metropolitana.

\section{Agradecimientos}

El desarrollo de esta investigación contó con el financiamiento de los proyectos 
PIP-11220130100407 (CONICET); PUE CONICET 2017-2022/22920170100036 y los aportes del Arq. Víctor Hugo Jiménez (Jefe Departamento de Ordenamiento Territorial, Municipalidad de Godoy Cruz).

\section{Referencias}

APEC. 2013. Building Code, Regulations and Standards. Minimum, Mandatory and Green. Reports. CTI Sub-Fora \& Industry Dialogues Groups, Sub-Committee on Standards and Conformance(SCSC). AsiaPacific Economic Cooperation. Recuperado el 30 de mayo, 2019, de https://www.apec. org/Publications/2013/08/APEC-BuildingCodes-Regulations-and-StandardsMinimum-Mandatory-and-Green

CAMMESA. 2019a. Balance Energético. Descargas de Informes. Varios. Informe Anual 2017. Compañía Administradora del Mercado Mayorista Eléctrico Sociedad Anónima. Recuperado el 21 de junio, 2019, de http://portalweb.cammesa.com/ memnet1/Pages/descargas.aspx

CAMMESA. 2019b. Máximos históricos de potencia y energía. Compañía Administradora del Mercado Mayorista Eléctrico Sociedad Anónima. Recuperado el 21 de junio, 2019, de http://portalweb. cammesa.com/default.aspx

EMESA. 2019. Inversores. Energía Hidroeléctrica. Empresa Mendocina de Energía Socidad Anónima. Recuperado el 21 de junio, 2019, de https://emesa.com.ar/ inversores-2/\#_electrica

Enker, Robert y Morrison, Gregory. 2017. "Analysis of the transition effects of buildings codes and regulations on the emergence of a low carbon residential building sector". Energy and Buildings, 156 (1): 40-50. Recuperado el 30 de mayo, 2019, de https:// doi.org/10.1016/j.enbuild.2017.09.059

INDEC. 2010. Censo Nacional de Población, Hogares y Viviendas 2010. Instituto Nacional de Estadísticas y Censos. Recuperado de https://www.indec.gob.ar/

Iwaro, Joseph y Mwasha, Abraham. 2010. "A review of building energy regulation and policy for energy conservation in developing countries". Energy Policy, 38 (12): 7744-
7755. Recuperado el 31 de mayo, 2019, de https://doi.org/10.1016/j.enpol.2010.08.027

Ley Nacional 27.191. 2015. Modificación al Régimen de Fomento Nacional para el uso de Fuentes Renovables de Energía destinada a la Producción de Energía Eléctrica. Recuperado de http://www.infoleg.gob.ar

Ley Provincial 8.999. 2017. Plan Provincial de Ordenamiento Territorial (PPOT). Recuperado de https://www. legislaturamendoza.gov.ar

Ley Provincial 4.886. 1983. Disposición de suelos en la zona oeste del Gran Mendoza. Recuperado de https://www. legislaturamendoza.gov.ar

Ley Provincial 4.341. 1979. Régimen para el loteo o fraccionamiento de terrenos. Recuperado de https://www. legislaturamendoza.gov.ar

Morales, Jorge.; Arboit, Mariela y Cucchietti, César. 2017. "Situación del consumo energético reciente en el ambiente construido del área metropolitana de Mendoza". V Jornadas Nacionales y I Internacionales de Investigaciones Interdisciplinarias Regionales. Enfoques para la historia.

Ordenanza 3.944. 2018. Construcciones sustentables. Capítulo U.II.4.1.4 U.II.4.1.5. Municipalidad de Ciudad de Mendoza. Recuperado de https://www. ciudaddemendoza.gov.ar

Ordenanza 4.947. 2003. Zonificación y usos del suelo. Municipalidad de Godoy Cruz. Recuperado de https://www.godoycruz.gob. ar

Ordenanza 5.924. 2011. Indicadores urbanos. Municipalidad de Godoy Cruz. Recuperado de https://www.godoycruz.gob.ar

Ordenanza 6.134. 2012. Corazón de manzana. Municipalidad de Godoy Cruz. Recuperado de https://www.godoycruz.gob.ar

Ordenanza 6.538. 2016. Descuento en aforo y bonificación en tasa municipal. Municipalidad de Godoy Cruz. Recuperado de https://www.godoycruz.gob.ar

Ordenanza 6.589. 2016. Mayor aprovechamiento del suelo urbano. Municipalidad de Godoy Cruz. Recuperado de https://www.godoycruz.gob.ar

Ordenanza 6.613. 2016. Alturas máximas en ejes residenciales especiales. Municipalidad 
de Godoy Cruz. Recuperado de https:// www.godoycruz.gob.ar

Ordenanza 6.646. 2017. Modificatoria de 6.538/16. Municipalidad de Godoy Cruz. Recuperado de https://www.godoycruz.gob. ar

Ordenanza 6.664. 2017. Programa municipal de promoción y fomento de energías limpias. Municipalidad de Godoy Cruz. Recuperado de https://www.godoycruz.gob.ar

Ordenanza 6.876. 2018. Plan Municipal de Ordenamiento Territorial (PMOT). Recuperado de https://www.godoycruz.gob. ar

Ordenanza 8.757.2011.Aspectos higrotérmicos y demanda energética de las construcciones. Municipalidad de Rosario. Recuperado de https://www.rosario.gob.ar

Pan, Wei y Garmston, Helen.2012. "Compliance with building energy regulations for newbuild dwellings". Energy, 48 (1): 11-22. Recuperado el 4 de junio, 2019, de https:// doi.org/10.1016/j.energy.2012.06.048

Pedro, Joao; Meijer, Frits y Visscher, Henk. 2010. "Technical building regulations in EU countries: a comparison of their organization and formulation". Recuperado el 4 de junio, 2019, de https://www.researchgate.net/ publication/260980505. 
Anexo de Tablas

Tabla 1. Urbanización Parque.

\begin{tabular}{|c|c|c|c|c|c|c|c|}
\hline \multirow{2}{*}{$\begin{array}{c}\text { Parcelas } \\
\text { Tamaño }\end{array}$} & \multirow{2}{*}{$\begin{array}{c}\text { Ocupa- } \\
\text { ción } \\
\text { máx }\end{array}$} & \multirow{2}{*}{$\begin{array}{c}\text { Constru } \\
\text { cción } \\
\text { máx }\end{array}$} & \multirow{2}{*}{$\begin{array}{c}\mathrm{H} \\
\text { máx }\end{array}$} & \multicolumn{3}{|c|}{ Retiros } & \multirow{2}{*}{$\begin{array}{c}\text { Separación } \\
\text { Torres }\end{array}$} \\
\hline & & & & RF & RL & RP & \\
\hline$<1000 \mathrm{~m}^{2}$ & 0,35 & 1 & $10 \mathrm{~m}$ & $4 \mathrm{~m}$ & \multicolumn{2}{|c|}{$1 / 3 \mathrm{~h}(\min 3 \mathrm{~m})$} & - \\
\hline$>1000 \mathrm{~m}^{2}$ & 0,35 & 1,5 & $10 \mathrm{~m}$ & $4 \mathrm{~m}$ & \multicolumn{2}{|c|}{ 1/3h (mín 3m) } & 1/3h (mín 6m \\
\hline $\begin{array}{c}\text { Vivienda } \\
\text { Multifa- } \\
\text { miliar en } \\
\text { Torre } \\
\end{array}$ & 0,16 & 1 & $10 \mathrm{~m}$ & $8 \mathrm{~m}$ & \multicolumn{2}{|c|}{$\begin{array}{c}\text { 1/3h (mín } \\
\text { 10m) }\end{array}$} & - \\
\hline
\end{tabular}

Tabla 2. Club de Campo.

\begin{tabular}{|c|c|c|c|c|c|c|c|}
\hline \multirow{2}{*}{$\begin{array}{l}\text { Uso de } \\
\text { Parcelas }\end{array}$} & \multirow{2}{*}{$\begin{array}{c}\text { Ocupa- } \\
\text { ción } \\
\text { máx }\end{array}$} & \multirow{2}{*}{$\begin{array}{c}\text { Constru } \\
\text { caión } \\
\text { máx }\end{array}$} & \multirow{2}{*}{ máx } & \multicolumn{3}{|c|}{ Retiros } & \multirow{2}{*}{$\begin{array}{c}\text { Separación } \\
\text { Torres }\end{array}$} \\
\hline & & & & $\mathrm{RF}$ & RL & $\mathrm{RP}$ & \\
\hline- & 0,35 & 1 & $10 \mathrm{~m}$ & $5 \mathrm{~m}$ & \multicolumn{2}{|c|}{ 1/3h (mín 3m) } & - \\
\hline \multicolumn{8}{|c|}{ Propiedad Horizontal } \\
\hline Exdusivo & 0,35 & 1 & $10 \mathrm{~m}$ & $5 \mathrm{~m}$ & \multicolumn{2}{|c|}{ 1/3h (mín 3m) } & - \\
\hline $\begin{array}{l}\text { Común } \\
\text { Privativas }\end{array}$ & 0,1 & 0,2 & - & \multicolumn{3}{|c|}{ 1/3h (mín 6m) } & 1/3h (mín 6m) \\
\hline
\end{tabular}

Tabla 3. Usos en zonas de la Ley.

\begin{tabular}{|c|c|c|c|c|}
\hline \multirow[t]{2}{*}{ Zonas } & \multirow{2}{*}{$\begin{array}{c}\text { Uso } \\
\text { Dominante }\end{array}$} & \multirow{2}{*}{$\begin{array}{c}\text { Uso } \\
\text { Condicionado }\end{array}$} & \multicolumn{2}{|c|}{$\begin{array}{c}\text { Densidad } \\
\text { Poblacional } \\
\end{array}$} \\
\hline & & & mín & máx \\
\hline 9: Parque Sur & Recreacional & - & - & - \\
\hline 10: Noroeste & \multirow[b]{2}{*}{ Residencial } & \multirow{2}{*}{$\begin{array}{l}\text { Comercial, } \\
\text { institucional, } \\
\text { recreacional, } \\
\text { industrial de } \\
\text { apoyo urbano }\end{array}$} & \multirow{2}{*}{$\begin{array}{c}100 \\
\text { hab } / \text { ha } \\
\text { (por } \\
\text { proyecto) }\end{array}$} & \multirow{2}{*}{$\begin{array}{l}250 \\
\text { hab/ha } \\
\text { (por } \\
\text { proyecto) }\end{array}$} \\
\hline 11: Suroeste & & & & \\
\hline $\begin{array}{l}\text { 12: Recupera- } \\
\text { ción Ripieras }\end{array}$ & \multicolumn{2}{|c|}{$\begin{array}{l}\text { sujeto a estudio especial por } \\
\text { estudio habitadional }\end{array}$} & - & - \\
\hline 13: Resto & \multicolumn{2}{|c|}{$\begin{array}{c}\text { Rural, recreacional, turístico, } \\
\text { cem enterios parque, urbani- } \\
\text { zación parque y dub de campo }\end{array}$} & - & $\begin{array}{c}175 \\
\text { hab/ha }\end{array}$ \\
\hline
\end{tabular}

Tabla 4. Indicadores urbano-edilicios en el municipio de Godoy Cruz.

\begin{tabular}{|c|c|c|c|c|c|c|}
\hline \multicolumn{7}{|c|}{ Indicadores urbano-edilicios de Godoy Cruz } \\
\hline Dens & & Factor de & Factor de & Altura bas: & | & eso \\
\hline Poblacional: & Parcela: & Ocupación del & Ocupación Total & Imen & más & e la \\
\hline cantidad de habitantes & superficie & Suelo (FOS): & (FOT): codiente & ibriendo el & $(\mathbf{H m}$ & lín \\
\hline lad de & indivisa del & $\operatorname{coc}$ & entre la superficie & perî & vert & edi \\
\hline su & terreno & ierta en & aubierta & desde & total & $\operatorname{los}$ \\
\hline aspira el & propuesto por & PB y la superficie total & superficie total de & hasta una al & alcanzar & del \\
\hline & & & & & edificaciones. & terreno. \\
\hline
\end{tabular}

Tabla 5. Zonas e Indicadores Urbanos.

\begin{tabular}{|c|c|c|c|c|c|c|c|c|c|c|c|c|c|c|c|}
\hline \multirow{3}{*}{ 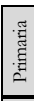 } & \multirow{3}{*}{ Zona } & \multicolumn{14}{|c|}{ Indicadores Urbanos } \\
\hline & & \multirow{2}{*}{$\begin{array}{c}\text { Densidad } \\
\text { Poblacional } \\
\end{array}$} & \multirow{2}{*}{\multicolumn{2}{|c|}{ Parcelas Tamaño }} & \multicolumn{2}{|c|}{ FOS } & \multicolumn{2}{|c|}{ FOT } & \multirow{2}{*}{\begin{tabular}{c|}
$\mathrm{H}$ \\
máxima
\end{tabular}} & \multirow{2}{*}{\begin{tabular}{|c|}
$\mathrm{h}$ basa- \\
mento
\end{tabular}} & \multirow{2}{*}{$\begin{array}{c}\text { h máx } \\
\text { fachada }\end{array}$} & \multicolumn{3}{|c|}{ Retiros } & \multirow{2}{*}{$\begin{array}{c}\text { Separación } \\
\text { Torres } \\
\end{array}$} \\
\hline & & & & & mín & máx & mín & máx & & & & $\mathrm{RF}$ & RL & $\mathrm{RP}$ & \\
\hline \multirow{6}{*}{ 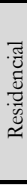 } & \multirow{3}{*}{$\begin{array}{c}\mathrm{R} 1, \mathrm{R} 2, \mathrm{R} 3, \mathrm{R} 4 \mathrm{y} \\
\text { R5 }\end{array}$} & \multirow{3}{*}{$\begin{array}{c}\text { Baja } \\
(100 \text { a } 250 \\
\text { hab/ha) }\end{array}$} & \multicolumn{2}{|c|}{100 a $500 \mathrm{~m}^{2}$} & - & 0,70 & - & 2,10 & \multirow{3}{*}{$\begin{array}{c}\mathrm{h} \text { basa- } \\
\text { mento }\end{array}$} & \multirow{6}{*}{$\begin{array}{c}\text { Optati- } \\
\text { vo. Varía } \\
\text { según a } \\
\text { calle. } \\
\text { h máx: } \\
12 \mathrm{~m} .\end{array}$} & \multirow{6}{*}{ - } & \multirow{6}{*}{\multicolumn{2}{|c|}{$\mathrm{R}=3+\mathrm{H} / 10 \geq 5 \mathrm{~m}$}} & \multirow{6}{*}{$\begin{array}{c}\mathrm{RP} \\
\text { Especial } \\
5+\mathrm{H} / 10 \\
\geq 7 \mathrm{~m}\end{array}$} & \multirow{6}{*}{$\begin{array}{c}\text { Retiro } \\
\text { Posterior } \\
\text { Especial } \\
5+\mathrm{H} / 10 \geq \\
7 \mathrm{~m}\end{array}$} \\
\hline & & & $501 \mathrm{a}$ & $1000 \mathrm{~m}^{2}$ & - & 0,65 & - & 1,95 & & & & & & & \\
\hline & & & +1 & $1001 \mathrm{~m}^{2}$ & - & 0,60 & - & 1,80 & & & & & & & \\
\hline & \multirow{3}{*}{ R6 } & Media & 100 & $500 \mathrm{~m}^{2}$ & - & 0,70 & - & 4,20 & \multirow{3}{*}{$18 \mathrm{~m}$} & & & & & & \\
\hline & & $(250$ a 400 & $501 \mathrm{a}$ & $1000 \mathrm{~m}^{2}$ & - & 0,65 & - & 3,90 & & & & & & & \\
\hline & & hab/ha) & +1 & $1001 \mathrm{~m}^{2}$ & - & 0,60 & - & 3,60 & & & & & & & \\
\hline \multirow{4}{*}{ 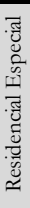 } & \multirow{4}{*}{$\begin{array}{c}\text { Ejes } \\
\text { Residenciales } \\
\text { Especiales }\end{array}$} & & 100 & $500 \mathrm{~m}^{2}$ & - & 0,75 & - & 7,50 & & & & & $\mathrm{R}=$ & & \\
\hline & & & $501 \mathrm{a}$ & $1000 \mathrm{~m}^{2}$ & - & 0,70 & - & 7,00 & $30 \mathrm{~m}$ & Optati- & & $\geq 7 \mathrm{~m}$ & $3+\mathrm{H} / 10$ & $\geq 7 \mathrm{~m}$ & Retiro \\
\hline & & Media & +1 & $1001 \mathrm{~m}^{2}$ & - & 0,65 & - & 6,50 & & vo. Varía & & & $\geq 5 \mathrm{~m}$ & & Posterior \\
\hline & & $\begin{array}{c}(250 \text { a } 400 \\
\text { hab/ha) }\end{array}$ & 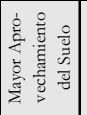 & $\begin{array}{c}100 \mathrm{a} 500 \mathrm{~m}^{2} \\
501 \mathrm{a} 1000 \mathrm{~m}^{2} \\
+1001 \mathrm{~m}^{2}\end{array}$ & $\begin{array}{l}- \\
- \\
-\end{array}$ & \begin{tabular}{l|l|}
0,75 \\
0,70 \\
0,65
\end{tabular} & $\begin{array}{l}- \\
- \\
-\end{array}$ & $\begin{array}{l}12,00 \\
11,00 \\
10,00\end{array}$ & $50 \mathrm{~m}$ & $\begin{array}{c}\text { segun a } \\
\text { calle. } \\
\text { h máx: } \\
12 \mathrm{~m} .\end{array}$ & - & $\mathrm{R}=$ & $\mathrm{H} / 10 \geq$ & $7 \mathrm{~m}$ & $\begin{array}{c}\text { Especial } \\
5+\mathrm{H} / 10 \geq \\
7 \mathrm{~m}\end{array}$ \\
\hline $\mathbb{8}$ & & Media & 100 & $500 \mathrm{~m}^{2}$ & - & 0,75 & - & 4,50 & & & & & & & \\
\hline$y_{y}^{\prime}$ & $\mathrm{RM}$ & $(250$ a 400 & $501 \mathrm{a}$ & $1000 \mathrm{~m}^{2}$ & - & 0,70 & - & 4,20 & $18 \mathrm{~m}$ & vo. Varía & & & & & Posterior \\
\hline .] & & hab/ha) & +1 & $1001 \mathrm{~m}^{2}$ & - & 0,65 & - & 3,90 & & según a & & $R=$ & & Especial & Especin \\
\hline E & & Baja & 100 & $500 \mathrm{~m}^{2}$ & - & 0,70 & - & 2,10 & & calle. & - & $\mathrm{R}=$ & $e_{1}$ & $5+\mathrm{H} / 10$ & $\begin{array}{l}\text { Especial } \\
5+H / 10>\end{array}$ \\
\hline$\frac{0}{0}$ & RM 1 & $(100$ a 250 & $501 \mathrm{a}$ & $1000 \mathrm{~m}^{2}$ & - & 0,65 & - & 1,95 & h basa- & h máx: & & & & $\geq 7 \mathrm{~m}$ & $5+\mathrm{H} / 10=$ \\
\hline & & hab/ha) & +1 & $1001 \mathrm{~m}^{2}$ & - & 0,60 & - & 1,80 & & & & & & & \\
\hline & & Media & 100 & $500 \mathrm{~m}^{2}$ & - & 0,80 & - & 6,40 & & & & & & & \\
\hline & $\mathrm{CM}$ & $(250$ a 400 & $501 \mathrm{a}$ & $1000 \mathrm{~m}^{2}$ & - & 0,75 & - & 6,00 & $24 \mathrm{~m}$ & vo. Varía & & & & & Netror. \\
\hline & & hab/ha) & +1 & $1001 \mathrm{~m}^{2}$ & - & 0,70 & - & 5,60 & & según a & & $\mathrm{B}=3+\mathrm{H}$ & & Especial & Fspecin \\
\hline & & Alta & 100 & $500 \mathrm{~m}^{2}$ & - & 0,80 & - & 8,00 & & calle. & & $\mathrm{K}=3+1$ & $\mathrm{n}$ & $5+\mathrm{H} / 10$ & $\begin{array}{c}\text { Especial } \\
5+\mathrm{H} / 10>\end{array}$ \\
\hline & C & $(400$ a 600 & $501 \mathrm{a}$ & $1000 \mathrm{~m}^{2}$ & - & 0,75 & - & 7,50 & $30 \mathrm{~m}$ & h máx: & & & & $\geq 7 \mathrm{~m}$ & $H / 10=$ \\
\hline & & hab/ha) & +1 & $1001 \mathrm{~m}^{2}$ & - & 0,70 & - & 7,00 & & & & & & & \\
\hline & & Media & 100 & $500 \mathrm{~m}^{2}$ & - & 0,80 & - & 4,80 & & Optativo. & & & & $\mathrm{RP}$ & Retiro \\
\hline . 팽 & Centro Cívico & $(250$ a 400 & $501 \mathrm{a}$ & $1000 \mathrm{~m}^{2}$ & - & 0,75 & - & 4,50 & $18 \mathrm{~m}$ & $\begin{array}{c}\text { arita segun } \\
\text { a calle. }\end{array}$ & & $\mathrm{R}=3+\mathrm{H} /$ & $10 \geq 5 \mathrm{~m}$ & Especial & Posterior \\
\hline के & & hab/ha) & +1 & $1001 \mathrm{~m}^{2}$ & - & 0,70 & - & 4,20 & & \begin{tabular}{|c|}
$\mathrm{h} \max :$ \\
$12 \mathrm{~m}$.
\end{tabular} & - & & & $\geq 7 \mathrm{~m}$ & $5+\mathrm{H} / 10 \geq$ \\
\hline & Res. Cultural & - & & - & - & - & - & - & - & - & & - & - & - & - \\
\hline & Cementerio & - & & - & - & - & - & - & - & - & & - & - & - & - \\
\hline
\end{tabular}


Tabla 6. Densidad real por Distrito. Fuente: Ord. 6.876, 2018.

\begin{tabular}{|c|c|c|c|c|c|}
\hline Distrito & Superficie $\mathbf{k m}^{\mathbf{2}}$ & Superficie ha & Personas $/ \mathbf{k m}^{\mathbf{2}}$ & Personas / ha & Población \\
\hline Tortugas & 5 & 500 & $7.796,80$ & 77,97 & 38.984 \\
\hline Ciudad & 12 & 1.200 & $7.062,25$ & 70,62 & 84.747 \\
\hline Benegas & 3,8 & 380 & $5.968,16$ & 59,68 & 22.679 \\
\hline Sarmiento & 14 & 1.400 & $3.315,86$ & 33,16 & 46.422 \\
\hline San Francisco & 5,5 & 550 & $2.098,73$ & 20,99 & 11.543 \\
\hline San Vicente & 67 & 6.700 & 2,60 & 0,03 & 174 \\
\hline
\end{tabular}

Tabla 7. Indicadores para hmáx basamento $9 \mathrm{~m}$.

\begin{tabular}{|c|c|c|c|}
\hline \multirow{2}{*}{$\begin{array}{c}\text { Parcelas } \\
\text { Tamaño }\end{array}$} & FOS & FOT & \multirow{1}{*}{\begin{tabular}{c} 
F \\
\cline { 2 - 3 } máxima
\end{tabular}} \\
\hline 100 a $500 \mathrm{~m}^{2}$ & 0,70 & 2,81 & \\
501 a $1000 \mathrm{~m}^{2}$ & 0,65 & 2,61 & \multirow{2}{*}{$12 \mathrm{~m}$} \\
$+1001 \mathrm{~m}^{2}$ & 0,60 & 2,41 & \\
\hline
\end{tabular}

Tabla 8. Indicadores sectores 1 y 2 .

\begin{tabular}{|c|c|c|c|}
\hline \multirow{2}{*}{$\begin{array}{l}\text { Parcelas } \\
\text { Tamaño }\end{array}$} & FOS & FOT & \multirow{2}{*}{$\begin{array}{c}\mathrm{H} \\
\text { máxima }\end{array}$} \\
\hline & máx & máx & \\
\hline 100 a $500 \mathrm{~m}^{2}$ & 0,80 & 8,00 & \multirow{3}{*}{$30 \mathrm{~m}$} \\
\hline 501 a $1000 \mathrm{~m}^{2}$ & 0,75 & 7,50 & \\
\hline$+1001 \mathrm{~m}^{2}$ & 0,70 & 7,00 & \\
\hline
\end{tabular}

Tabla 9. Comparación de Zonificación en el AMM.

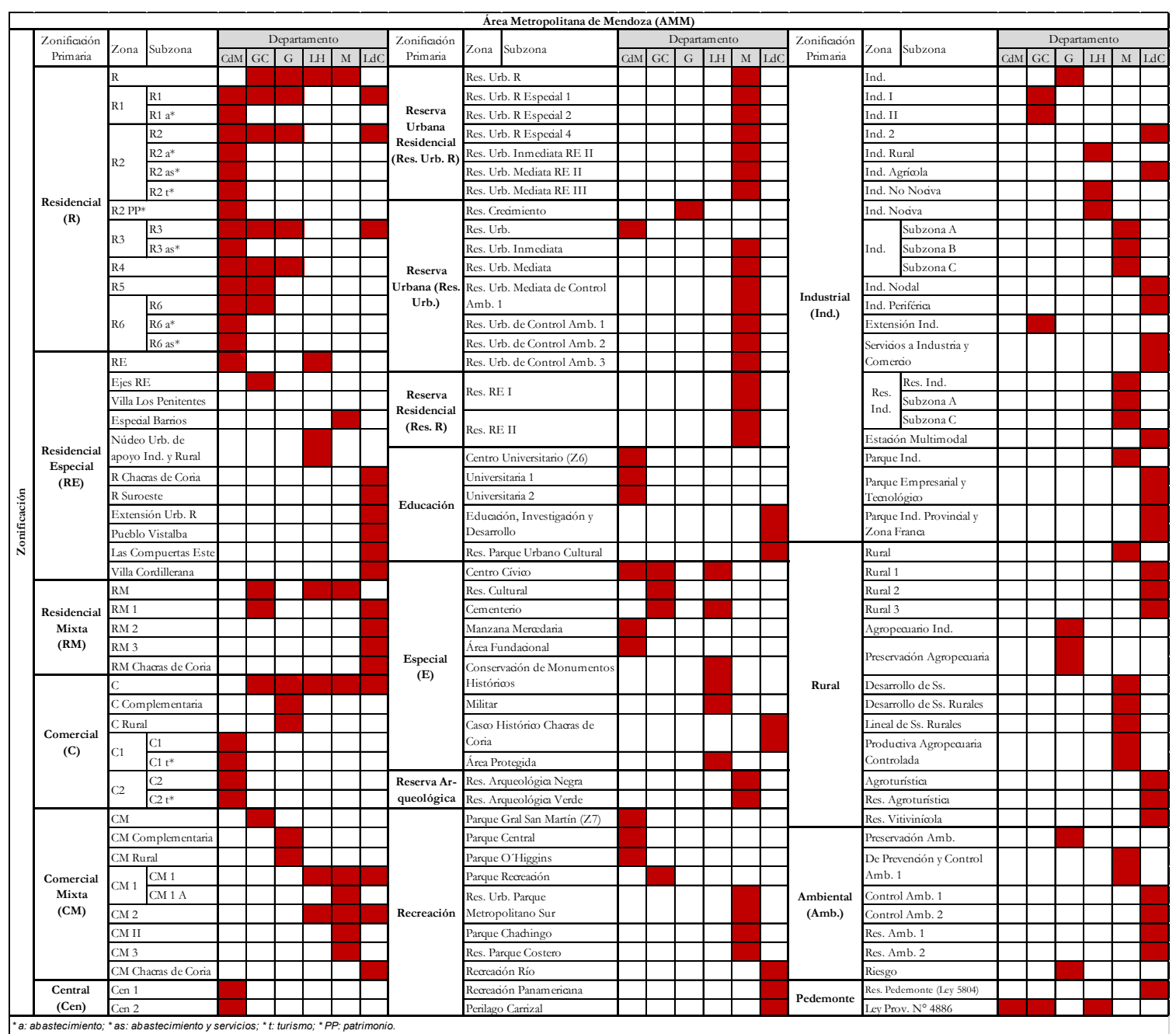


Tabla 10. Comparación de Indicadores en el AMM.

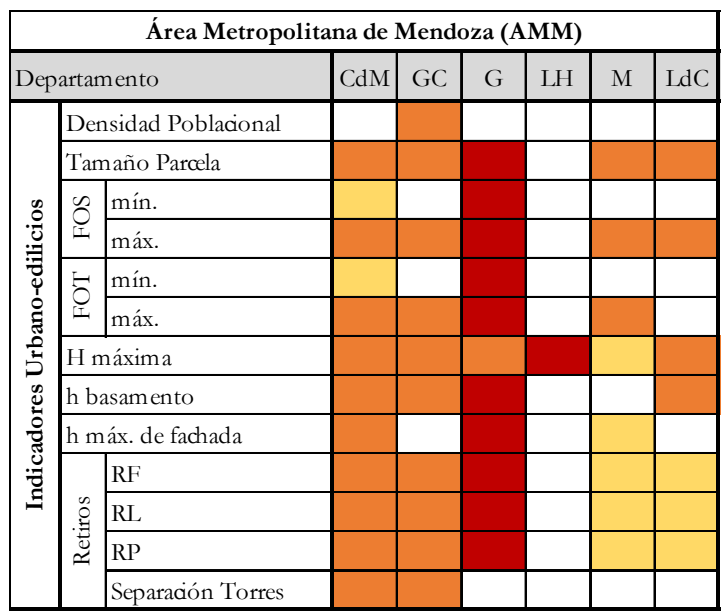

\section{REFERENCIAS}

En todas las zonas y subzonas

En la mayoría de las zonas y subzonas

En algunas zonas y subzonas

En ninguna ó prácticam ente ninguna zona y subzona

Tabla 12. Godoy Cruz. Aforo - Tasa. Descuento de Aforos

\begin{tabular}{|c|c|}
\hline Obras & Descuento \\
\hline$\leq 120 \mathrm{~m}^{2}$ & $60 \%$ \\
\hline $121 \mathrm{a} 500 \mathrm{~m}^{2}$ & $40 \%$ \\
\hline$\geq 501 \mathrm{~m}^{2}$ & $25 \%$ \\
\hline
\end{tabular}

Tabla 14. Ciudad de Mendoza. Sustentabilidad

\begin{tabular}{|c|c|}
\hline \multirow{3}{*}{ 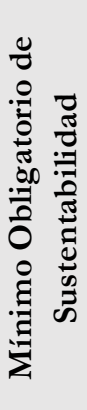 } & $\begin{array}{l}\text { Incorporar sistemas de } \\
\text { control solar exterior e } \\
\text { interior en el } 100 \% \text { de los } \\
\text { aventanamientos. }\end{array}$ \\
\hline & $\begin{array}{c}\text { Manejar valores de albedo } \\
\text { de } 0.8 \text { a } 1 \text { en superficies } \\
\text { exteriores horizontales. }\end{array}$ \\
\hline & $\begin{array}{l}\text { Crear áreas verdes con el } \\
\text { mínimo requerimiento } \\
\text { hídrico. }\end{array}$ \\
\hline
\end{tabular}

Tabla 11. Indicadores de Desarrollo Urbano Sustentable.

\begin{tabular}{|c|c|c|c|c|c|c|c|}
\hline \multicolumn{8}{|c|}{ Área Metropolitana de Mendoza (AMM) } \\
\hline \multicolumn{2}{|c|}{ Departamento } & $\mathrm{CdM}$ & GC & G & LH & M & LdC \\
\hline \multirow{3}{*}{ 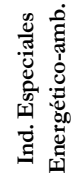 } & Corazón de Manzana & & & & & & \\
\hline & $\begin{array}{l}\text { Construcciones } \\
\text { Sustentables }\end{array}$ & & & & & & \\
\hline & Energías Limpias & & & & & & \\
\hline
\end{tabular}

\section{REFERENCIAS}

Obligatorios

Optativos por optención de un beneficio

Ninguno
Tabla 13. Godoy Cruz.Aforo - Tasa. BonificaciónTasa Municipal

\begin{tabular}{|c|c|c|}
\hline Obras & Descuento & Tiempo \\
\hline$\leq 120 \mathrm{~m}^{2}$ & $30 \%$ & \multirow{2}{*}{24} \\
\cline { 1 - 2 } $121 \mathrm{a} 500 \mathrm{~m}^{2}$ & $20 \%$ & \multirow{2}{*}{$\begin{array}{c}24 \\
\text { meses }\end{array}$} \\
\hline$\geq 501 \mathrm{~m}^{2}$ & $10 \%$ & \\
\hline
\end{tabular}

Tabla 15. Ciudad de Mendoza. Parámetros

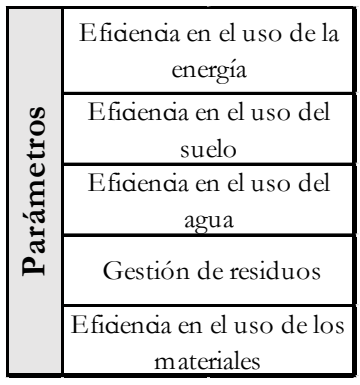

